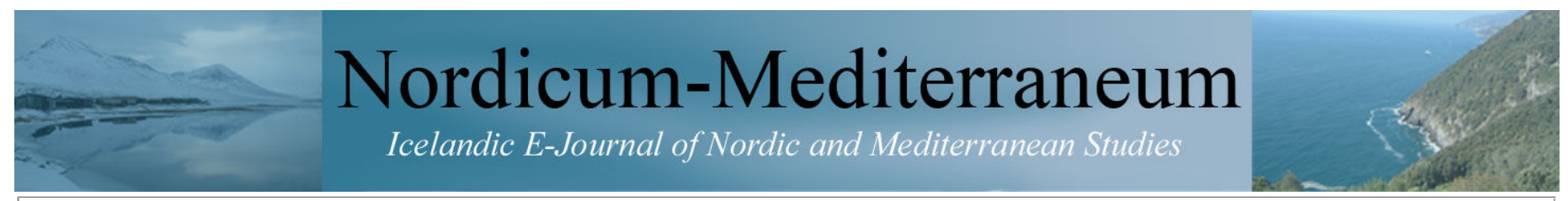

March 2008

Volume 3, Number 1

\title{
Il valore della testimonianza - The Value of Testimony
} by Nicla Vassallo

The following text is from my book Teoria della conoscenza published by Laterza some years ago. I have chosen to offer it here, in memory of my friend and colleague Flavio Baroncelli, for three basic reasons. First of all, more than twenty years ago, Flavio offered to be the supervisor of my graduate thesis on an epistemological theme in modern philosophy. I have not forgotten that possibility and have in recent times come to reflect upon both the type of epistemology and the philosophers that were in modern philosophy. I have not forgotten that possibility and have in recent times come to reflect upon both the type of epistemology and the philosophers that were
beloved by Flavio. The text which follows these introductory remarks is a testament to this. Secondly, when he knew that I was interested in the epistemology of testimony, Flavio urged me to go on, because of the importance of that topic not only for general epistemology, but also for many other branches of philosophy. Thirdly, on the occasion of the publication of Teoria della conoscenza, Flavio participated on the round table in which it was presented to the public and had something like this to say: "I read the whole book with the same breath, as it was a detective story". In his memory, I cannot but hope to write other "detective stories"

Il testo che segue è tratto dal mio volume Teoria della conoscenza uscito da Laterza qualche anno or sono. Ho scelto di riproporlo qui, in memoria dell'amico e collega Flavio Baroncelli per tre ragioni fondamentali. Innanzitutto, più di vent'anni fa, mi era stata offerta da Flavio la possibilità di laurearmi con lui su un tema epistemologico della filosofia moderna. Non avendolo dimenticato, sono tornata recentemente a riflettere (come attesta quanto segue) proprio su un tipo di epistemologia e su autori cari a Flavio. In secondo luogo, più recentemente, quando ha saputo che mi stavo interessando di epistemologia della testimonianza Flavio mi epistemologia e su autori cari a Flavio. In secondo luogo, più recentemente, quando ha saputo che mi stavo interessando di epistemologia della testimonianza Flavio mi
ha sollecitato a continuare, evidenziando che l'importanza dell'epistemologia in questione travalica i confini della stessa epistemologia generale. In terzo luogo, quando ha partecipato, in occasione dell'uscita del volume, alla tavola rotonda in cui si è discusso di Teoria della conoscenza, Flavio ha detto qualcosa come: "L'ho letto tutto d'un fiato. Come se fosse un libro giallo". In sua memoria, non posso che sperare di scrivere altri libri gialli.

Si possono rilevare ovvie connessioni tra le diverse fonti conoscitive. Per accennare a un solo esempio, al fine di poter ricordare che p, dobbiamo prima aver percepito che p, o inferito che p, o essere stati consapevoli che p o aver avuto una testimonianza che p. E' sulla testimonianza che intendiamo ora soffermarci di più, per due motivi principali: in primo luogo, a differenza delle altri fonti conoscitive, è stata epistemologicamente poco indagata e vedremo che le sono state ingiustamente preferite altre fonti conoscitive, quali la percezione e il ragionamento; in secondo luogo, gli umani sono esseri sociali che hanno sviluppato e continuano a sviluppare un sofisticato sistema di mutuo scambio d'informazioni e di conoscenze. Molto spesso le nostre credenze, o le nostre conoscenze, sono dovute alle parole degli altri, all'altrui testimonianza, cioè derivano non solo dalla familiare conversazione faccia a faccia, ma anche dalla conversazione telefonica, da quanto ascoltiamo alla radio, dai documenti scritti, dagli scambi via e-mail, da internet. Se ci affidassimo solo alla percezione, alla memoria, alla consapevolezza, alla ragione, ma non alla testimonianza, conosceremmo decisamente meno di quanto supponiamo di conoscere: non potremmo conoscere gli eventi del passato, o quegli eventi del presente che sono molto lontani da noi, o quelle teorie scientifiche che sono al di fuori del raggio delle nostre competenze. Non potremmo conoscere neanche fatti molto banali che diamo per assodati, quali che la terra è rotonda, abbiamo un cervello, la nostra data di nascita è il tal giorno, mese e anno, i nostri genitori biologici sono Tizio e Caio. La stessa scienza non costituisce un territorio alieno dalla testimonianza. Molte teorie che uno scienziato accetta dipendono da quanto altri scienziati sostengono. Lo stesso vale per i dati, poiché spesso uno scienziato non esegue personalmente tutti gli esperimenti necessari in cui i dati vengono acquisiti, né l'analisi dei medesimi: queste sono operazioni che, il più delle volte, richiedono troppo tempo per essere eseguite da un singolo soggetto cognitivo. Inoltre, le ricerche scientifiche vengono sempre più portate innanzi da gruppi, e non da singoli: è una necessità dovuta al fatto che la conoscenza dei singoli non è sufficiente per condurre esperimenti.

Se è vero che ogni osservazione è "theory-laden" ("carica di teoria”), cioè è sempre dipendente dalla teoria, anche le osservazioni dello scienziato sono legate alla testimonianza: è attraverso quest'ultima che per la maggior parte vengono trasmesse le teorie. La psicologia della Gestalt e Wittgenstein (1953) sono gli antesignani della nozione di "theory-laden", mentre Hanson (1958) l'ha sviluppata compiutamente. Egli si chiede se Keplero, che crede che la terra si muova attorno al sole, e Tycho Brahe, che crede il contrario, vedano il medesimo evento quando osservano il sole sorgere. Secondo Hanson, rispetto al sorgere del sole, Keplero e Tycho Brahe si trovano nella medesima condizione di noi tutti quando osserviamo le immagini ambigue e vediamo cose diverse (in una stessa immagine possiamo vedere un'anatra o un coniglio; in un'altra immagine, una donna anziana o una giovane avvenente, e così via). Hanson giunge a concludere che l'osservazione è un'impresa "theory-laden", condizionata dalla conoscenza anteriore di quanto si osserva e influenzata anche dal linguaggio, che viene impiegato per esprimere ciò che sappiamo, senza l'ausilio del quale saremmo in grado di riconoscere davvero poco come conoscenza. E le teorie ci vengono per la maggior parte trasmesse attraverso la testimonianza.

Non è, peraltro, necessario affrontare nei dettagli una qualche scienza per comprendere la nozione di "theory-laden"; è sufficiente considerare una semplice proposizione osservativa dell'esperienza quotidiana, come "Aiuto, Virginia sta annegando nel mare!". Questa affermazione dipende evidentemente da una teoria, teoria che implica l'esistenza di una persona chiamata Virginia, la quale possiede la proprietà di annegare, e l'esistenza di un oggetto chiamato mare in cui si può annegare. Inoltre, l'espressione "aiuto" trasmette una richiesta di soccorso, legata al fatto che una persona chiamata Virginia stia annegando e si trovi, quindi, di fronte all'evento di potersi fare male o, addirittura, di morire. L'affermazione, pertanto, è sì legata alla percezione, ma dipende, a sua volta, da una teoria complessa e composta da proposizioni, o credenze, o conoscenze, in chi parla o in chi ascolta, relative all'esistenza delle persone, delle cose, delle proprietà, delle richieste e degli eventi. Queste credenze vengono acquisite in tenera età e vengono, per la maggior parte, trasmesse per testimonianza dai genitori o dagli insegnanti.

Il termine "testimonianza" evoca comunemente l'immagine di un palazzo di giustizia. Qui la testimonianza è spesso legata al ragionamento. Se sono un giudice non accetto tout court una testimonianza, ma valuto il testimone - sia che egli sia un testimone oculare, sia che egli riporti una teoria scientifica (balistica o psichiatrica, e così via). Colloco la testimonianza nell'ambito del processo e la soppeso, alla luce della mia conoscenza di sfondo, per accogliere ciò che sembra vero solo sulla base di un'ottica ampia. Cosicché l'accettazione di una testimonianza viene qui a dipendere da un ragionamento: date le premesse che (supponiamo) il testimone sembra credibile e che le sue affermazioni collimano con quanto so a proposito del caso giudiziario in questione, giungo a credere con ragione ad esse.

Sembra, allora, che la testimonianza sia una fonte conoscitiva secondaria rispetto al ragionamento, poiché lo presuppone. Nella vita quotidiana, tuttavia, le credenze prodotte dalla testimonianza hanno carattere inferenziale solo quando vengono riferite proposizioni che ci paiono strane. Vediamo un caso. Si immagini di incontrare una persona $Y$, a noi ignota, e che questa ci riferisca di essere stata ad un convegno dove il professore X ha mostrato forti segni di squilibrio mentale. Dato che conosciamo bene X e non abbiamo mai dubitato della sua salute mentale, sospendiamo il giudizio sulla testimonianza, o rimaniamo increduli. Man, mano che il racconto di Y procede, ragioniamo e siamo in grado di valutare la credibilità della sua testimonianza per poi accettarla o di rifiutarla. In genere, però, quando ci vengono riferite proposizioni che non ci risultano anomale, la testimonianza non richiede nostre inferenze. Quando amici fidati ci testimoniano una certa proposizione e non abbiamo ragione di pensare che la proposizione in questione sia al di là delle loro competenze, normalmente la crediamo punto e basta. Più, in generale, quando le persone ci testimoniano qualcosa, noi semplicemente le crediamo, se quanto ci testimoniano non è in conflitto con qualche nostra credenza o conoscenza precedente. Consideriamo il nostro tipico comportamento durante una situazione comune della vita quotidiana: un viaggio. Dobbiamo recarci a Barcellona. Il nostro agente di viaggio ci vende un biglietto aereo e noi crediamo a quanto egli ci riferisce circa l'ora di partenza e di arrivo dell'aeroplano. Una volta giunti a destinazione, in albergo, il cameriere ci telefona, annunciandoci che il pranzo sarà servito dalle 13.30 alle 15.30 , e ci affidiamo a quanto egli afferma. Dopo pranzo, vogliamo vedere alcune opere di Antoni Gaudì. Leggiamo la nostra guida turistica e non dubitiamo che essa riporti la verità circa gli indirizzi della Casa Batllò, della Pedrera, della Sagrada Familia. Da quando siamo partiti fino alla nostra consultazione della guida, abbiamo accettato diverse testimonianze, senza alcuna inferenza. Tale accettazione è un'importante caratteristica psicologica della nostra vita quotidiana.

Perché allora l'epistemologia della testimonianza non gode di un ampio riconoscimento? Non tanto perché l'epistemologia viene tradizionalmente distinta dalla psicologia, ma piuttosto perché la giusta valutazione della testimonianza è stata fortemente ostacolata da una concezione individualista del soggetto cognitivo. Descartes, considerato da molti il fondatore della teoria della conoscenza in senso moderno, è convinto ad esempio che il soggetto cognitivo debba essere libero e rispettabile sotto il profilo epistemico, e possa esserlo solo se non fa conto sulla testimonianza. A capo dei razionalisti moderni, Descartes, come i suoi successori, conferisce priorità alla ragione e al ragionamento su qualsiasi altra fonte conoscitiva. Gli empiristi dissentono e privilegiano la percezione. Non tengono, tuttavia, la testimonianza in maggiore considerazione: nel Saggio sullintelletto umano, Locke afferma che i progressi conoscitivi reali si hanno solo nella misura in cui noi stessi consideriamo la verità e percepiamo il mondo, e non nella misura in cui accettiamo l'altrui testimonianza.

L'assoluta svalutazione della testimonianza, operata da Descartes e Locke, va incontro a qualche difficoltà. Partiamo dal programma cartesiano e dal suo solipsismo metodologico: al soggetto cognitivo è imposto di contare solo sulle proprie evidenze interiori al fine di sconfiggere lo scettico e di conseguire quella conoscenza certa dalla quale derivare la dimostrazione filosofico-razionale dell'esistenza di Dio e del mondo esterno. All'inizio delle Meditazioni metafisiche Descartes rigetta, innanzitutto, la testimonianza della Bibbia, per, poi, disfarsi di tutto quanto ha appreso nel corso della sua esistenza e ripartire da zero per fondare una conoscenza e una scienza ferme e durevoli. Si può, però, rilevare che tale mossa non gli è consentita: per scrivere le Meditazioni, egli deve contare ancora sul linguaggio e, pertanto, non può rinunciare alle conoscenza di esso: una conoscenza che egli, come del resto 
noi tutti, ha sicuramente appreso attraverso la testimonianza, intenzionale o non, di altri soggetti. Possiamo apprendere, per esempio, la parola "gatto", sia osservando che c'è un gatto ogniqualvolta qualcuno pronuncia la parola "gatto" (testimonianza inintenzionale), sia, più frequentemente, perché qualcuno ci dice "questo è un gatto", quando c'è un gatto, o perché ci dice "il gatto è un animale a quattro zampe che miagola" (testimonianza intenzionale). Se Descartes intende scrivere le Meditazioni, egli è costretto ad ammettere l’importanza della testimonianza quale fonte conoscitiva: non ammettendola, egli non può scriverle - potrebbe, forse, pensarle, ma non dal punto di vista di chi sostiene che non è possibile pensare se non in qualche linguaggio.

Veniamo ora a Locke e agli empiristi. Contro la priorità da loro conferita alla percezione, se ci si limita all'osservazione visiva, sussiste il problema, già citato, che ogni osservazione è "theory-laden": non è, pertanto, consentito privilegiare la percezione, senza privilegiare anche la testimonianza. Certo, a favore degli empiristi, si può notare il fatto che accade di rifiutare una testimonianza in virtù delle nostre individuali osservazioni. Tornando al nostro esempio, una volta a Barcellona, vogliamo recarci a visitare La Pedrera. Chiediamo indicazioni a un passante il quale risponde: "Dritti, avanti verso nord per Passeig de Gràcia, sulla sinistra". Proseguendo nella direzione indicataci, osserviamo, ad un certo punto, La Pedrera sulla destra e questa nostra osservazione ci conduce a ricusare la testimonianza secondo la quale essa avrebbe dovuto trovarsi sulla sinistra. La nostra ricusazione è però ribadiamolo - a favore degli empiristi, solo se ogni osservazione non è "theory-laden", ossia, non è dipendente, in ultimo, dalla testimonianza. E, d'altronde, contro gli empiristi, si può ribadiamolo - a favore degli empiristi, solo se ogni osservazione non è "theory-laden", ossia, non è dipendente, in ultimo, dalla testimonianza. E, d'altronde, contro gli empiristi, si può
addurre il fatto speculare che ci capita di rifiutare le nostre osservazioni in virtù di una testimonianza, come (per esempio) nel caso in cui stiamo attraversando in traghetto l'arcipelago della Maddalena e alla nostra affermazione "Quella è l’isola di Budelli", il marinaio replica "No, vi sbagliate, quella è l'isola di Razzoli": reagiamo rigettando la nostra affermazione insieme all'osservazione che la sostiene e accettiamo la testimonianza del marinaio.

Gli estremismi di un certo empirismo a proposito della testimonianza non convincono Hume che dichiara: «Non c'è una specie di ragionamento più comune, più utile e anche necessario alla vita umana, di quello che si ricava dalla testimonianza e dai resoconti di testimoni oculari e di spettatori» (1748, 1957, p. 124). A parte il fatto che, come vi è visto, la testimonianza non è necessariamente legata a un qualche ragionamento, questa dichiarazione rivaluta l'importanza di essa. Tuttavia, Hume, da buon empirista, non le attribuisce il medesimo status che alla percezione; a quest'ultima viene attribuita una posizione in ogni caso privilegiata: egli propone di giustificare la nostra accettazione della testimonianza riscontrando, proprio attraverso l'osservazione, che i fatti corrispondano a quanto ci viene testimoniato. Uno dei problemi ovvi di questa proposta è che le testimonianze riportano fatti riscontrando, proprio attraverso l'osservazione, che i fatti corrispondano a quanto ci viene
che, per lo più, risultano alla maggior parte di noi inaccessibili sotto il profilo osservativo.

A dissentire da Hume è Reid che, in An Inquiry into Human Mind (1764), sottolinea, contro la concezione individualistica del soggetto cognitivo, il nostro essere creature sociali, predisposte ad affidarci alla testimonianza naturalmente, e non in virtù delle ragioni, o giustificazioni, di cui disponiamo: se dovessimo basarci su queste ultime non crederemmo, né conosceremmo, un centesimo di quanto ci viene detto. Secondo Reid, il principio della veracità (siamo propensi a dire la verità) e il principio della credulità (siamo propensi a credere agli altri) sono sempre all'opera in noi, a meno che non intervengano altri fattori, quali, possiamo supporre, l'accorgerci che il testimone è un mentitore, o il possesso d'evidenza contraria rispetto a quanto ci viene testimoniato, o il prendere atto che la testimonianza è in conflitto con le nostre credenze. La considerazione di questi altri fattori introduce, in ogni caso, il problema della giustificazione. In termini reidiani possiamo affermare che, per essere giustificati a credere una certa proposizione p riferitaci da un certo testimone $\mathrm{T}$, requisiti minimi sono il nostro comprendere $\mathrm{p}$, il non aver alcuna valida ragione di dubitare della sincerità di T, il non disporre d'alcuna buona evidenza contro p, o d'alcuna credenza in minimi sono il nostro comprendere $\mathrm{p}$, il non aver alcuna valida ragione di dubitare della sincerità di $\mathrm{T}$, il non disporre d'alcuna buona evidenza contro $\mathrm{p}$, o d'alcuna credenza in
conflitto con p. A questo si può aggiungere il requisito che impone di non aver alcuna valida ragione per pensare che non sia giustificata la credenza di $\mathrm{T}$ relativamente a p, o il requisito più forte che impone di aver una valida ragione per pensare che sia giustificata la credenza di $\mathrm{T}$ relativamente a $\mathrm{p}$.

Posti davanti alla domanda "perché credi che una certa proposizione p sia vera?" o di fronte alla domanda "come fai a sapere che una certa proposizione p è vera", abbiamo potenzialmente a disposizione diverse risposte: (i) percepisco che p, (ii) ricordo che p, (iii) inferisco che p, (iv) sono consapevole che p, (v) mi viene testimoniato che p. (i) può verificarsi di fronte a proposizioni del tipo "il gatto è sul divano": la fonte conoscitiva corrispondente è la percezione. Si può prospettare (ii) in presenza di proposizioni del tipo "il mio trisavolo si chiamava Napoleone Vassallo": la fonte conoscitiva corrispondente è la memoria. Si può delineare (iii) nel caso di proposizioni del tipo "Tutti gli uomini sono mortali e Socrate è un uomo, quindi Socrate è mortale" (inferenza deduttiva) o "Il corvo è è nero, il corvo 2 è nero, il corvo 3 è nero, il corvo è è vero, tutti i corvi sono neri" (inferenza induttiva): la fonte conoscitiva corrispondente è la ragione o il ragionamento. Si può ottenere (iv) di fronte a tutte le proposizioni che riguardano la conoscenza di sé (es. "penso di essere felice", "immagino un mare color smeraldo"; "sento di amarla"): la fonte conoscitiva corrispondente è la percezione interna o la consapevolezza o l'introspezione. Infine, proposizioni del tipo "Clinton è stato presidente degli Stati Uniti" o "Ipazia era una filosofa, astronoma e matematica greca" o " 2 + 2 = 4 " o "Marte è un pianeta del nostro sistema solare" possono dare adito alla risposta (v): la fonte conoscitiva corrispondente è la testimonianza. Le prime quattro fonti conoscitive sono individuali, mentre la quinta è sociale: questo significa solo che la conoscenza ottenuta per mezzo della testimonianza necessita dell'interazione sociale e non che è il sociale, piuttosto che l'individuale, a conoscere veramente.

(Tratto da Nicla Vassallo, Teoria della conoscenza, Laterza, Roma-Bari, 2003. Si ringrazia l'editore Laterza per averne gentilmente concesso la riproduzione.)

Nicla Vassallo (Imperia, 1963), autorevole specialista di filosofia della conoscenza, è attualmente Professore Ordinario di Filosofia Teoretica presso l'Università di Genova e Visiting Professor di Epistemologia presso l'Università Vita-Salute San Raffaele di Milano. Book Review Editor della rivista Epistemologia, membro dell'Advisory Board dell'European Journal of Analytic Philosophy, dell'Institute for Scientific Methodology, di L\&PS: Logic and Philosophy of Science, della rivista Iride: Fïlosofia e discussione pubblica, fa parte del Consiglio scientifico del Festival della scienza, del Festival per l'Economia Interculturale, dell'Osservatorio Nazionale sulla Salute della Donna. Tra le sue numerose pubblicazioni scientifiche (che hanno meritato sessantacinque menzioni su The Philosopher's Index) ricordiamo alcuni volumi in italiano: Teoria della conoscenza (Laterza, Roma-Bari 2003, seconda edizione 2008) in qualità di autrice, Filosofia delle donne (Laterza, Roma-Bari 2007, seconda edizione 2007) in qualità di co-autrice, Filosofia delle conoscenze (Codice Edizioni, Torino 2006) in qualità di curatrice, Filosofia della comunicazione (Laterza, Roma-Bari 2005; seconda edizione 2006) in qualità di co-curatrice.

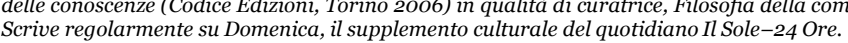

